\title{
Hybrid refractive holographic single vision spectacle lenses
}

\author{
Jannik M. Trapp ${ }^{1,2^{*}}$ (D), Toufic G. Jabbour ${ }^{3}$, Gerhard Kelch ${ }^{4}$, Thomas Pertsch ${ }^{2,5}$ and Manuel Decker ${ }^{1}$
}

\begin{abstract}
In this contribution, we investigate hybrid single vision spectacle lenses (SVSLS) consisting of holographic optical elements (HOEs) embedded into a refractive lens. We evaluate the performance of two examples of hybrid SVSLs in terms of their distributions of spherical error (SPH error), astigmatic error (AST error) and transverse chromatic error (CE) over the lens surface, simulating the optical performance for the patient's rotating eye. We find that, particularly for high prescription values, hybrid SVSLS outperform their purely refractive counterparts in terms of CE with the additional benefit of reducing the lens thickness. As such, we show that hybrid refractive, holographic designs can be a viable alternative to purely refractive SVSLS for high prescription SVSLS.
\end{abstract}

Keywords: Ophthalmic lenses, Spectacle lens design, Vision, Holography, Holographic optical element, Diffraction

\section{Introduction}

Single vision spectacle lenses (SVSLs) are refractive lenses created to correct refractive errors of the human eye such as myopia, hyperopia and astigmatism by providing the required spherical power $(\mathrm{SPH})$ and/or astigmatic power (AST) [1-6] depending on the patient's prescription. However, in SVSLs with a high prescription of SPH or AST, dispersion induced transverse chromatic error (CE) can limit the imaging performance to a perceivable degree which may even be disturbing to the patient. Therefore, it is highly desirable to keep CE below a certain threshold over the patient's field of gaze. The only practical way to reduce $\mathrm{CE}$ in conventional refractive SVSLs is to switch to a spectacle lens material with a higher Abbe number i.e. less dispersion. However, lens materials (polymers and glass) with a higher Abbe number typically have a lower refractive index [7]. Lenses with low refractive index, in turn, typically need a higher front surface curvature than high index lenses for the same prescription to achieve the same optical performance. This leads to larger edge thickness in negative SPH SVSLs and larger center thickness in positive SPH SVSLs. For the patients, curvature and thickness

\footnotetext{
* Correspondence: jannik.trapp@zeiss.com

${ }^{1}$ Corporate Research and Technology, Carl Zeiss AG, Carl Zeiss Promenade

10, 07745 Jena, Germany

${ }^{2}$ Institute of Applied Physics, Abbe Center of Photonics, Friedrich Schiller

University Jena, Albert-Einstein-Str. 15, 07745 Jena, Germany

Full list of author information is available at the end of the article
}

increases can be highly undesirable for aesthetic reasons when fitting the SVSLs into standard frames and because of weight increases causing discomfort. An achromatic doublet consisting of a negative $\mathrm{SPH}$ and a positive SPH lens with adjusted Abbe numbers is also not a viable option for SVSLs with reduced CE due to the large thickness and weight increases associated with using two lenses. Therefore, it is very attractive to find a novel method of reducing CE in SVSLs, particularly for high SPH and/or AST prescriptions, without increasing the lens thickness or compromising the target distributions of SPH and AST.

In this contribution, we investigate how $\mathrm{CE}$ reduction can be achieved in hybrid SVSLs consisting of holographic optical elements (HOEs) embedded into a refractive lens. The basic idea for achieving CE correction is to combine a refractive lens with positive dispersion and HOEs that are characterized by negative dispersion for the same sign of SPH. This means that a hybrid SVSL, in which the prescription value of SPH is achieved by contributions from HOEs and the refractive lens, automatically has less dispersion-induced CE than a single refractive lens of the same SPH prescription. Importantly, although CE correction via diffractive-refractive dispersion compensation per se is well known [8-11], this concept cannot be straightforwardly applied to SVSLs since they require supporting the desired functionality over a large range of gaze directions at the same 
time. We demonstrate that this requirement can be met with the use of HOEs. We also show that HOEs can deliver optical functions such as a certain SPH or AST in a holographic film of less than $100 \mu \mathrm{m}$ thickness. This also results in smaller lens curvatures i.e. flatter and thinner lenses are possible.

The main challenge of using HOEs is in SVSLs is to design them so that their optical function in terms of $\mathrm{SPH}$ and AST takes the desired prescription values for all gaze directions of the eye. At the same time, the use of HOEs is inherently connected with additional requirements that need to be fulfilled for the $\mathrm{HOE}$ to be of practical use such as high diffraction efficiency or minimal grating dispersion. The main challenge, then, is to find an optimized design that delivers the required prescription values of SPH and AST while fulfilling all the said requirements. For this purpose, we apply a dedicated optimization toolchain based on our previous work that achieves a broadband median HOE diffraction efficiency of 0.88 [12, 13].

In the following, we first review general SVSL design basics and present exemplary refractive SVSLs that serve as a benchmark for our hybrid SVSL designs. We then investigate hybrid SVSL designs with the same prescription as the benchmark SVSLs and evaluate their performance with respect to each other.

\section{Background}

The main goal of SVSL design is to provide the prescription values of SPH and AST, which correct the refractive errors of the eye of the patient. The refractive errors of the eye and therefore the prescription are independent of gaze direction. However, as the eye rotates between different gaze directions, the pupil moves and different parts of the SVSL are used. Therefore, the SVSL needs to correct the refractive errors of the eye for all gaze directions at the same time. From an optics design perspective, this is known as pupil walking [14] since the eye pupil is the system aperture. Figure $1 \mathrm{a}$ and $\mathrm{b}$ exemplarily show two gaze directions of an eye looking at a SVSL to illustrate this aspect.

In practice, it is not possible for an SVSL to exactly meet the desired prescription values for all gaze directions. Therefore, SVSLs are typically designed so that the desired prescription is met in the center of the lens, which corresponds to the gaze direction of looking straight ahead as depicted in Fig. 1a. For off-centered gaze directions, the requirement to meet the desired prescription is relaxed, however, deviations from the prescription values of SPH and AST (SPH error and AST error, respectively) need to be minimized to ensure the optimal function of the SVSL. In the following, SPH and AST prescriptions are usually given as the reciprocal of the respective back focal lengths in diopters $(\mathrm{dpt}=1 / \mathrm{m})$ with reference to the vertex sphere $[15,16]$. This means that the curvatures of the lens need to be chosen so that the back focal lengths of the blue ray bundle correspond to the desired prescription. When considering another gaze direction, e.g. looking downwards as shown in Fig. 1b, it can be seen by geometry that the green ray bundle does not see the same prescription as the blue ray bundle. When evaluating SVSL designs, we calculate the actual SPH and AST from the back vertex focal lengths of the ray bundles limited by the pupil for different gaze directions. We assume a pupil diameter of $2 \mathrm{~mm}$ for our calculations, which is an appropriate value for vision in bright daylight. In the following, we calculate deviations from the prescribed SPH and AST for a fine sampling of gaze directions and plot the results as SPH error and AST error over the lens surface. The typical goal of refractive SVSL design is to provide the correct prescription value of SPH and AST at least at the optical center of the lens and minimal SPH error and AST error for the rest of the lens. The exact balance of SPH error and AST error is a trade-off and can be tailored to the patient's or prescribing optometrist's preferences.

Our main motivation here is to investigate benefits of hybrid SVSLs in terms of CE compensation. In literature, CE of refractive SVSLs is usually calculated using
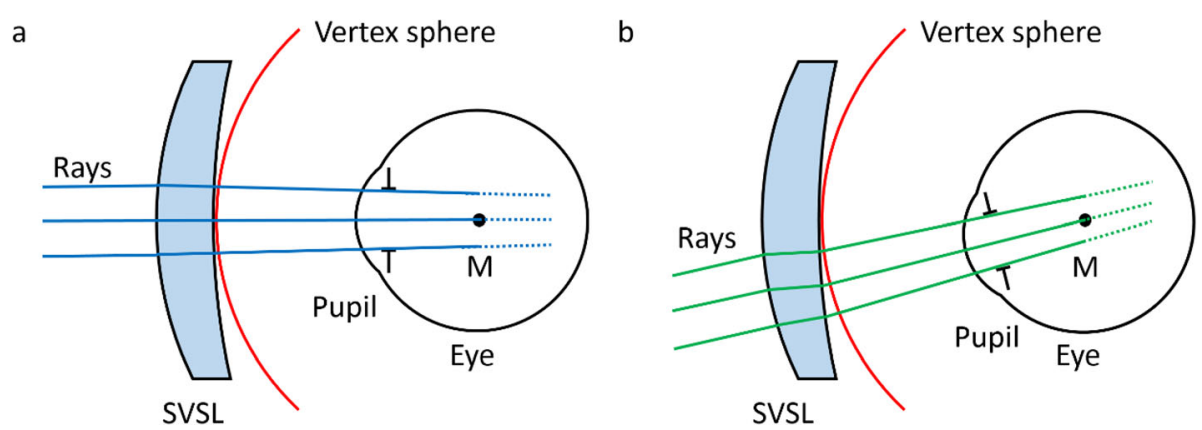

Fig. 1 Illustration of two sample gaze directions (a) and (b) of an eye using a spectacle lens. The aim of optical design of spectacle lenses is to correct the refractive errors of the optics of the eye for all possible gaze directions. These refractive errors such as SPH or AST are defined using the back focal lengths of the lens with reference to the vertex sphere 
an approximate formula based on the Abbe number of the lens material [17]. The relevant human perception threshold for CE derived with this formula is reported as $0.12 \mathrm{~cm} / \mathrm{m}$ [17]. In practice, this means that an observer would see $0.24 \mathrm{~cm}$ thick color fringes around the edges of a black object placed on a white screen in $2 \mathrm{~m}$ distance.

In our process, we then define an alternative definition of CE to cover holographic and hybrid SVSLs as well since they cannot be described solely by an Abbe number and the approximate formula is not applicable. Instead, we calculate the CE of SVSLs as the distance between red and blue rays at $620 \mathrm{~nm}$ and $430 \mathrm{~nm}$ wavelength in the pupil of the eye, respectively. Because we are interested in the relative improvements of hybrid SVSLs over refractive SVSLs of the same prescription, we normalize the CE results to the performance of a refractive SVSL at the given prescription that we calculate before.

To establish an understanding of the performance and limits of refractive SVSLs, we designed several sample SVSLs for the prescription of $-4 \mathrm{dpt}$ SPH and $0 \mathrm{dpt}$ AST with a lens diameter of $60 \mathrm{~mm}$, which exhibit color fringes up to $0.29 \mathrm{~cm} / \mathrm{m}$ or a normalized CE of unity on the lens edge. The perception threshold of $0.12 \mathrm{~cm} / \mathrm{m}$ color fringes then corresponds to a normalized CE of 0.4. All SVSLs are made from a standard SVSL polymer with refractive index 1.59 and Abbe number 41.11. First, we consider a spherical lens as used in tabletop optics. Optimizing the radii of the spherical lens to achieve the required prescription at the lens center $(X=0 \mathrm{~mm}, \mathrm{Y}=0 \mathrm{~mm})$ yields the distributions of SPH error, AST error and normalized CE shown in Fig. 2a-c. While the prescription value is achieved in the lens center, SPH error and AST error, increase towards the edge of the lens. This is expected for geometrical arguments for oblique gazes discussed earlier for Fig. 1b. CE is also increasing towards the edge of the lens since the oblique gaze hits lens areas with increasing prismatic effect, according to Prentice's rule [8]. This prismatic effect directly causes $\mathrm{CE}$. The maximal $\mathrm{CE}$ value is 1 due to our normalization. We note that, in practice, such a spherical lens is not recommended as its SPH error and AST error can be further reduced by using an asphere (ASPH).

In a next step, we designed a state-of-the-art SVSL with one spherical and one aspherical surface. The ASPH can be optimized to reduce SPH error or AST error. Typically, the balance between SPH error and AST error is a compromise based on the individual needs of the patient. Figure 2d-f and Fig. 2g-i present SVSL designs in which an ASPH was used to reduce AST error (d)-(f) and SPH error (g-i), respectively. Both SVSLs have almost identical CE since ASPHs are known to have minimal influence on CE. This, however, also means that ASPHs cannot be used to achieve CE corrected performance. The SVSLs in Fig. 2 will be used as benchmark SVSLs later to evaluate holographic or hybrid SVSLs optimized for the same prescription.

\section{Methods}

In this section, we discuss the challenges of including HOEs in SVSLs and present our design method for holographic and hybrid SVSLs. First, we want to discuss the challenges of using diffractive optics such as HOEs in SVSLs. Since grating dispersion in HOEs is much higher $[8,18,19]$ than in refractive SVSLs of comparable $\mathrm{SPH}$, it is necessary to pre-compensate for a certain amount of dispersion to limit CE. We achieve this by using two HOEs in a tandem configuration as described in $[12,13]$. A further challenge is diffraction efficiency: On the one hand, the HOE needs to operate with high diffraction efficiency in a single diffraction order over the entire visible spectrum of light. On the other hand, a certain angular bandwidth needs to be supported with high diffraction efficiency to account for the continuous motion of the eye. In our optimization tool chain $[8,9]$, we use rigorous HOE simulations based on the Fourier Modal Method [20, 21] to identify a $\mathrm{HOE}$ parameter range, in which high diffraction efficiency over a large angular and wavelength bandwidth is achieved simultaneously. We then use these results as input to a raytracing based optical design tool chain to tailor the free HOE parameters and to achieve the desired prescription values in terms of SPH and AST for all gaze directions of the eye. Effectively, we represent each gaze direction as an optical system configuration with its own optical axis and then optimize the HOE parameters of all configurations simultaneously to provide a continuous distribution of grating periods and slant angles over the lens surface. The minimum SPH error, AST error, CE, the lens thickness as well as the optimum HOE parameter range for the grating period are used as merit functions for the optimization process.

\section{Results and discussion}

In this section, we compare the performance of refractive, holographic and hybrid SVSL designs. We investigate a purely holographic SVSL consisting of two HOEs embedded into a flat polymer sheet without curvature to establish an understanding on how the performance of the HOE differs from the refractive SVSLs introduced in Fig. 2. Afterwards, we investigate the performance of a hybrid system consisting of HOEs and a spherical lens to find SVSL designs with minimal CE. Finally, we investigate SVSLs consisting of HOEs and an aspherical lens with an ASPH on one surface (see Fig. 3). Our goal is to achieve new and better trade-offs between SPH error, AST error, CE and lens thickness, which are only accessible using the degrees of freedom provided by the HOEs. To allow easy comparison between color plots, most 

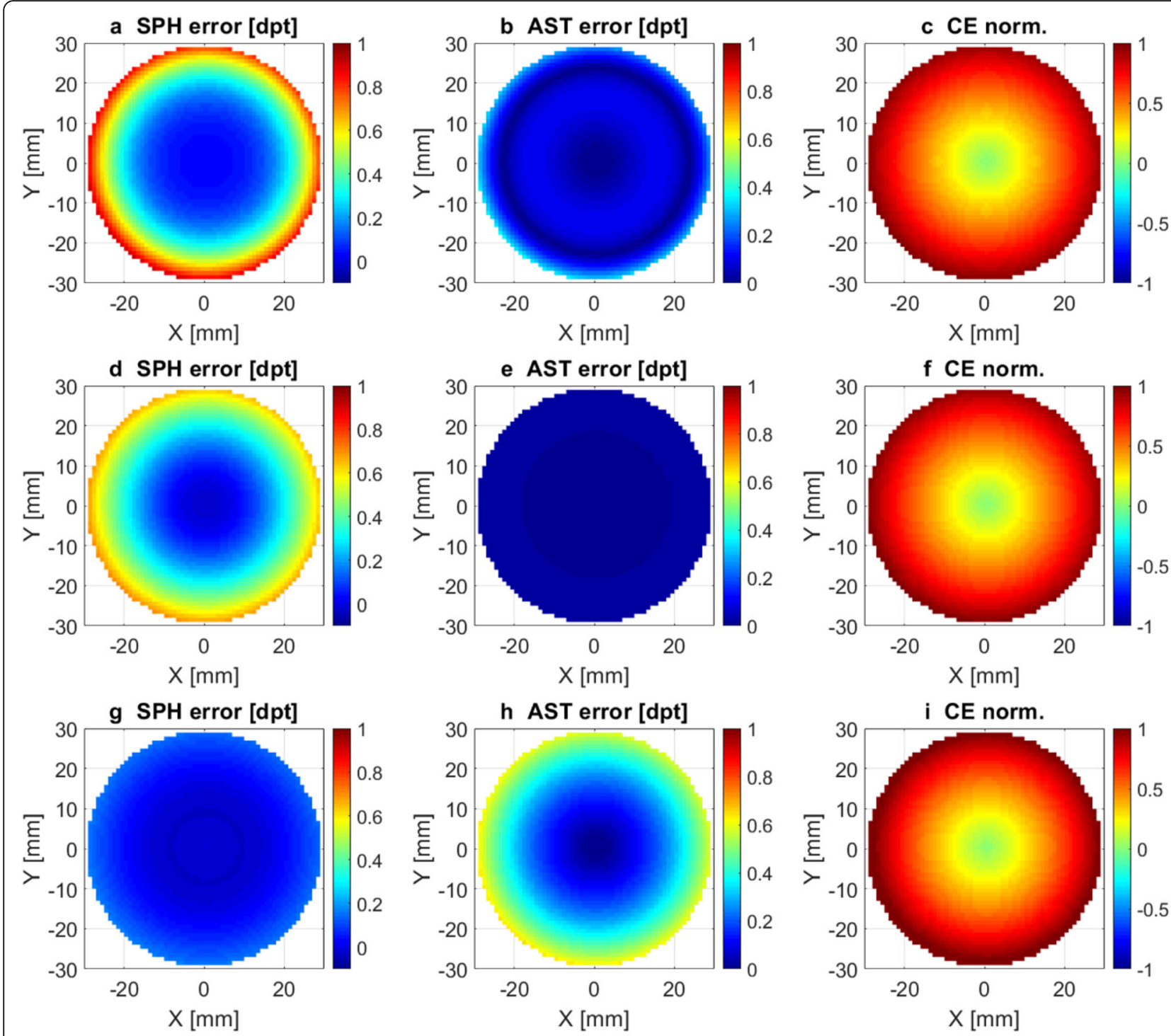

Fig. 2 Investigation of refractive SVSLs with prescription -4 dpt SPH and 0 dpt AST made from a standard SVSL polymer with refractive index 1.59 and Abbe number 41.11. a-c are the SPH error, AST error and CE of a spherical lens. ASPHs can be used to reduce AST error or SPH error as shown in $\mathbf{d}-\mathbf{f}$ and $\mathbf{g}-\mathbf{i}$, respectively. CE remains almost unaffected by the ASPH

SVSLs presented here are SVSLs designed for a target prescription of $-4 \mathrm{dpt}$ SPH and 0 dpt AST. We chose this prescription as it allows investigating relevant levels of AST error and CE. However, our findings are applicable to all types of SVSLs. To illustrate this, we also discuss a SVSL with target prescription of $-8 \mathrm{dpt}$ SPH and 0 dpt AST.

To establish an understanding on how the performance of purely holographic SVSLs differs from refractive ones, we design SVSLs consisting of two HOEs embedded into a flat sheet of a standard SVSL polymer with refractive index 1.59 and Abbe number 41.11 without curvature. These SVSLs are purely holographic SVSLs, since the contribution of the polymer sheets to the SPH and AST distribution of the SVSL is negligible. We optimize such a SVSL for a prescription of -4 dpt SPH and $0 \mathrm{dpt}$ AST to allow direct comparison with the refractive SVSLs shown in Fig. 2. The resulting distributions of SPH error, AST error and CE are shown in Fig. 4a-c. SPH error and AST error are plotted in the same color scale as in Fig. 2. We find that the SPH error of the holographic SVSL is qualitatively very similar to the one of the refractive SVSL shown in Fig. 2d. The maximum AST error, however, is up to $1 \mathrm{dpt}$ in the holographic SVSL as compared to up to $0.02 \mathrm{dpt}$ in the refractive SVSL. This increase in AST error can be attributed to our HOEs being not rotationally symmetric, which is a result of the deflection angle between the two 


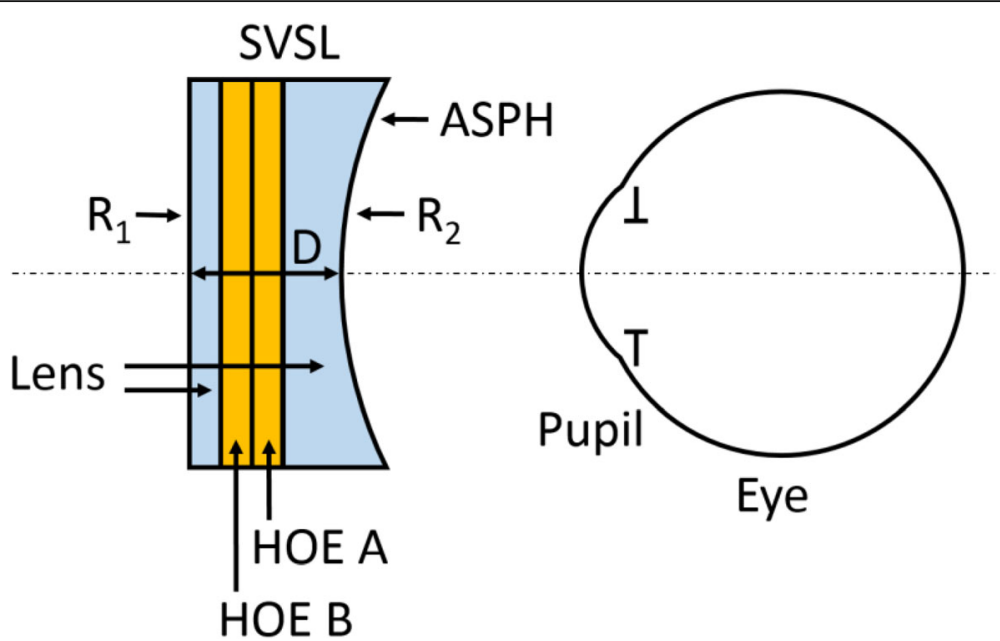

Fig. 3 Sketch of the discussed hybrid SVSLs. These SVSLs consist of a lens with front radius R1, back radius R2, center thickness D, two HOEs embedded into the lens and, in some cases, an ASPH on the backside of the lens

HOEs used in tandem [12, 13]. CE is plotted in a different scaling in Fig. 4c, because the CE shown here exceeds the CE of all refractive SVSLs by far. Notably, higher $\mathrm{CE}$ is to be expected since dispersion in HOEs is much more pronounced than in refractive lenses [22], which means that for a fixed SPH prescription a holographic lens such as the HOEs is expected to induce considerably more $\mathrm{CE}$ than their refractive counterparts. While the holographic SVSL shown here clearly is not an attractive design, it helps our understanding of the optical design problem.

Using the insights we gained from analyzing the holographic SVSL, we now design hybrid SVSLs. Designing hybrid SVSLs consisting of HOEs and refractive lenses requires extending our design method to include the lens geometry and material. The lens geometry is implemented via the center thickness, front and back radius and can include the coefficients of an ASPH placed on one of the refractive (front or back) surfaces. Figure 3 shows a sample geometry of a SVSL consisting of a lens with center thickness $\mathrm{D}$, front radius $\mathrm{R} 1$ and back radius R2, two HOEs embedded into the lens and an ASPH located on the backside of the lens. SVSLs like the one shown in Fig. 3 are hybrid SVSLs with refractive and holographic contributions to the overall optical function of SPH and AST. As known from technical optics, holographic and refractive contributions to SPH cause CE of opposite sign. This allows the design of CE-compensated optical systems.

First, we design hybrid SVSLs consisting of two HOEs embedded into a spherical lens. This means that different to the purely holographic SVSL discussed in the previous paragraph we now design a hybrid SVSL in which the holographic (HOE) and refractive (spherical lens) contributions of $\mathrm{CE}$ can compensate each other. The
SPH error, AST error and CE of a sample design of such a hybrid SVSL with the same prescription of $-4 \mathrm{dpt}$ $\mathrm{SPH}$ and $0 \mathrm{dpt}$ AST as the previous refractive SVSLs (Fig. 2) are shown in Fig. 4 (d)-(f). The performance of the hybrid SVSL is a combination between the performance of the holographic SVSL shown in Fig. 4a-c and the refractive SVSL shown in Fig. 2 (a)-(c). CE compensation in such hybrid SVSLs is typically achieved for large refractive and small holographic contributions to the overall SPH e.g. a 90:10 ratio. In our case, the final value is determined by an optical design optimization method, which also minimizes the AST error. As discussed earlier, the holographic contributions to SPH come with a higher AST error than the refractive ones. Therefore, our result is a compromise between introducing holographic $\mathrm{SPH}$ contributions for $\mathrm{CE}$ compensation while minimizing holographic SPH contributions to keep AST error low. Here, we reduced CE below the perception threshold of 0.4 . Since the SPH error is similar for the refractive and the hybrid SVSL, no changes are observed here.

The best SVSLs achievable with our design method are SVSLs consisting of HOEs and an aspherical lens with an ASPH, here on the backside. This is because the previous results have shown that ASPHs are the most potent way of reducing the SPH error and AST error distribution of SVSLs, while the CE induced by HOEs can cancel the $\mathrm{CE}$ induced by refractive components [8]. Therefore, we combine the two approaches. In the following, we investigate SVSLs consisting of HOEs embedded into aspherical lenses and evaluate their performance in terms of SPH error, AST error, CE and edge thickness. We design a SVSL with a prescription of $-4 \mathrm{dpt}$ and $0 \mathrm{dpt}$ AST. Our goal is to design a SVSL with a similar performance in terms of SPH error and AST error as the purely refractive benchmark SVSL 


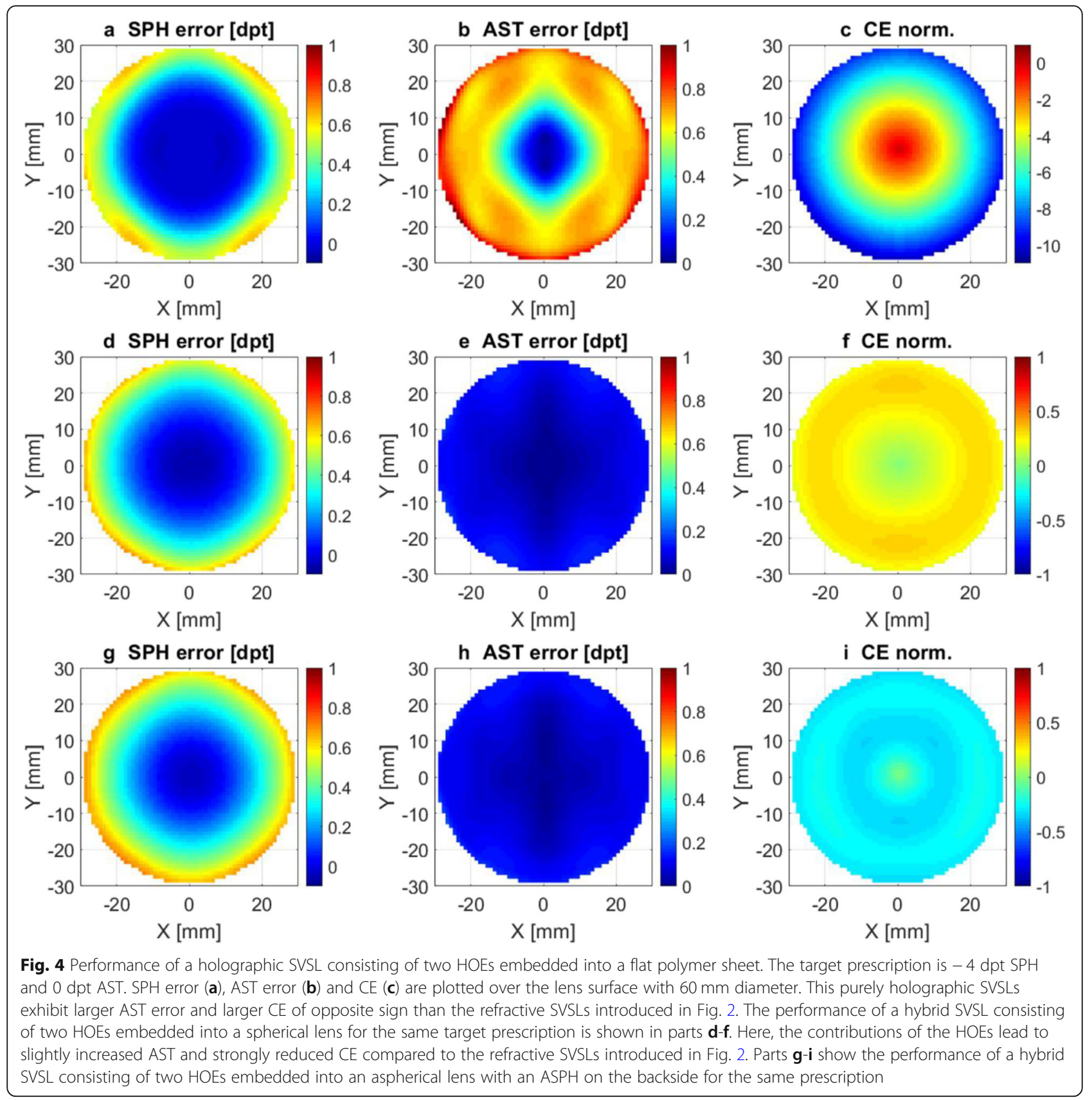

introduced in Fig. 2d-f along with lower CE and smaller edge thickness. We optimize a SVSL with an aspherical lens made of a standard SVSL polymer with refractive index of 1.59, two HOEs embedded into the lens and the ASPH placed on the backside of the lens. The resulting distributions of SPH error, AST error and CE are shown in Fig. 4g-i. Our sample design has a SPH error distribution comparable to the benchmark SVSL in Fig. 2d-f and a slight increase of maximum AST error to about 0.15 $\mathrm{dpt}$, which is close the lowest tolerance of $0.09 \mathrm{dpt}$ for spectacle lenses [23]. Its CE, however, is lower by a factor of more than 2.5 and the edge thickness is reduced from $4.5 \mathrm{~mm}$ to $3.8 \mathrm{~mm}$. It should be noted that in this example CE is not only reduced, but also negative. This is because if one aims to minimize the edge thickness of a negative SPH SVSL it is desirable to reduce the curvatures of the two lens surfaces. This can be done by increasing the amount of SPH contributed by the HOEs. The consequence is that $\mathrm{CE}$ is negative and AST is higher, because the larger the contribution of the HOEs the higher the resulting AST.

In a final step, we evaluate the benefits of our design method for SVSLs with a prescription of $-8 \mathrm{dpt}$ SPH and $0 \mathrm{dpt}$ AST as the practical relevance of CE compensation 
in SVSLs increases with the absolute SPH prescription. We design a refractive benchmark SVSL consisting of an aspherical lens made of a standard spectacle lens polymer with refractive index 1.73, Abbe number 32.15 and an ASPH on the backside. The resulting distributions of SPH error, AST error and CE are shown in Fig. 5a-c. CE is normalized as in the previous figures, so a $\mathrm{CE}$ of 0.4 corresponds to the perception threshold and a CE of 1 corresponds to the CE of a $-4 \mathrm{dpt}$ SPH refractive SVSL as shown in Fig. 2. Maximum CE is about 2.5 here and it is known from practice that the $-8 \mathrm{dpt} \mathrm{SPH}$ and 0 AST prescription SVSL exhibits color fringes, which are problematic for many patients.

We now design a hybrid SVSL with -8 dpt SPH in which mainly the $\mathrm{CE}$ is reduced without increasing the edge thickness and without increasing SPH and AST error notably above the lowest spectacle lens tolerance standard [14]. The distribution of SPH error, AST error and CE for our hybrid SVSL design are shown in Fig. 5d-f. We find that $\mathrm{SPH}$ error and AST error distributions are comparable to the benchmark SVSL shown in Fig. 5a-c while CE is significantly reduced by $60 \%$. As a result, the CE of the hybrid -8 dpt SVSL is approximately at the same level of the refractive SVSL with $-4 \mathrm{dpt}$ SPH prescription. In addition, the edge thickness of the hybrid -8 dpt SVSL is reduced by $0.2 \mathrm{~mm}$ to $5.5 \mathrm{~mm}$. It should be noted that we chose a front curvature of $700 \mathrm{~mm}$ for the hybrid SVSL, while is essentially flat. This means that even at high prescriptions, fabrication techniques that require flat HOEs are compatible with our approach.

\section{Conclusion}

We have designed hybrid refractive, holographic SVSLs with -4 and -8 dpt SPH with strongly reduced CE. For this purpose, we embedded two HOEs in tandem configuration in an aspherical refractive lens and optimized the design to provide minimum CE with negligible SPH error or AST error. We found that for the $-4 \mathrm{dpt}$ hybrid SVSL design the CE can be reduced below the perception threshold while at the same time reducing the lens thickness by $16 \%$, without compromising the desired distributions of SPH error and AST error. For the $-8 \mathrm{dpt}$ hybrid SVSL we achieved a CE reduction of around $60 \%$ while reducing the lens thickness by approximately $4 \%$.

Our findings show that by using a combined holographic, refractive lens design approach, particularly for high prescription SVSLs, it is possible to significantly reduce color fringes and, therefore, alleviate disturbances

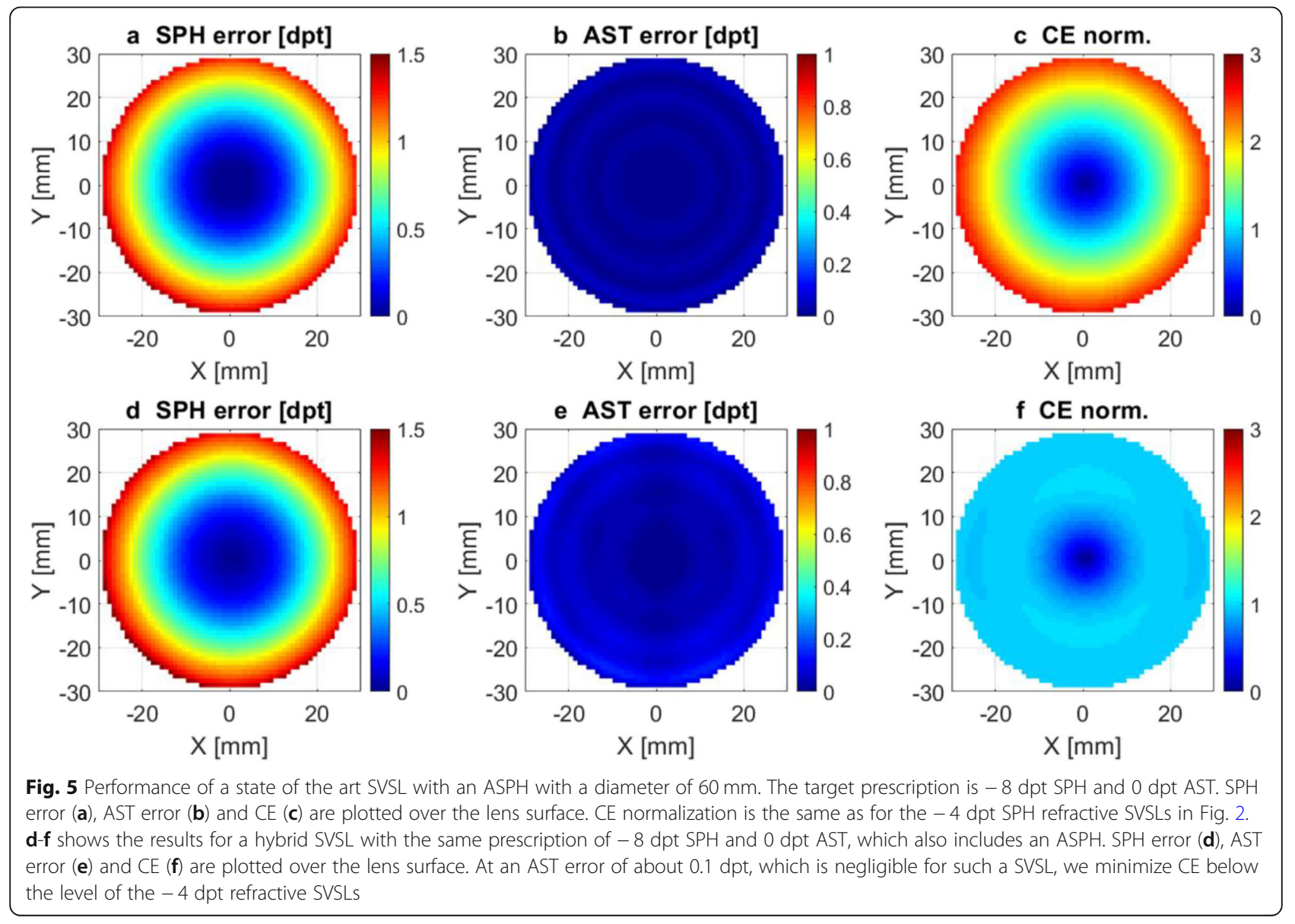


of a patient's vision with high prescription SVSLs. Furthermore, due to the reduction of the lens thickness, the resulting SVSLs are highly desirable since the reduced weight also reduces discomfort for the patient. Both examples clearly show that our design approach allows finding new and desirable SVSL designs with a significantly improved performance and wearability.

Manufacturing our hybrid SVSL designs requires advanced manufacturing techniques such as holographic printers [24-26] that are capable of creating arbitrary HOE configurations. Recent improvements in holographic materials suggest that our simulated diffraction efficiencies can be realized experimentally $[27,28]$.

\section{Acknowledgements}

Not applicable.

\section{Authors' contributions}

TP, TGJ and MD. designed and supervised the project. JMT. designed, implemented and evaluated the optical design tool chain with input on holography from TGJ. and input on commercial spectacle lens design by GK All authors discussed the results. JMT and MD wrote the manuscript with input and from all authors. All authors read and approved the final manuscript.

\section{Funding}

This project has received funding from the EU's Horizon 2020 research and innovation programme under the Marie Skłodowska-Curie grant agreement No 675745.

\section{Availability of data and materials}

Not applicable.

\section{Competing interests}

The authors declare that they have no competing interests.

\section{Author details}

${ }^{1}$ Corporate Research and Technology, Carl Zeiss AG, Carl Zeiss Promenade 10, 07745 Jena, Germany. ${ }^{2}$ Institute of Applied Physics, Abbe Center of Photonics, Friedrich Schiller University Jena, Albert-Einstein-Str. 15, 07745 Jena, Germany. ${ }^{3}$ Corporate Research and Technology, Carl Zeiss AG, Carl-Zeiss-Straße 22, 73447 Oberkochen, Germany. "Technology and Innovation, Carl Zeiss Vision GmbH, Turnstraße 27, 73430 Aalen, Germany. ${ }^{5}$ Fraunhofer Institute for Applied Optics and Precision Engineering, Albert-Einstein-Str. 7, 07745 Jena, Germany.

Received: 1 April 2019 Accepted: 5 June 2019

Published online: 18 June 2019

\section{References}

1. Gross, H., Blechinger, F., Achtner, B.: Handbook of Optical Systems, Volume 4, Survey of Optical Instruments. Wiley-VCH (2008)

2. Artal, P.: Handbook of Visual Optics, Volume Two: Instrumentation and Vision Correction. CRC Press (2017)

3. Atchison, D.A.: Third-order theory and aspheric spectacle lens design. Ophthalmic Physiol. Opt. 4, 179-186 (1984)

4. Atchison, D.A.: Spectacle lens design: a review. Appl. Opt. 31, 3579-3585 (1992)

5. Barbero, S.: Minimum tangential error ophthalmic lens design without multi-parametric optimization. Opt. Commun. 285, 2769-2773 (2012)

6. Jia, Z., Xu, K., Fang, F.: Measurement of spectacle lenses using wavefront aberration in real view condition. Opt. Express. 25, 22125-22139 (2017)

7. Gross, H.: Handbook of optical systems. In: Fundamentals of Technical Optics, vol. 1. Wiley-VCH (2005)

8. Stone, T., George, N.: Hybrid diffractive-refractive lenses and achromats. Appl. Opt. 27, 2960-2971 (1988)

9. Wood, A.: Design of infrared hybrid refractive-diffractive lenses. Appl. Opt. 31, 2253-2258 (1992)
10. Flores, A., Wang, M.R., Yang, J.J.: Achromatic hybrid refractive-diffractive lens with extended depth of focus. Appl. Opt. 43, 5618-5630 (2004)

11. Artigas, J.M., Menezo, J.L., Peris, C., Felipe, A., Díaz-Llopis, M.: Image quality with multifocal intraocular lenses and the effect of pupil size: comparison of refractive and hybrid refractive-diffractive designs. J Cataract Refract Surg. 33, 2111-2117 (2007)

12. J. Trapp, M. Decker, J. Petschulat, T. Pertsch, and T. G. Jabbour, "Holographic progressive lenses," in SPIE Optical Engineering + Applications (SPIE2018), p. 7

13. Trapp, J.M., Decker, M., Petschulat, J., Pertsch, T., Jabbour, T.G.: Design of a 2 diopter holographic progressive lens. Opt. Express. 26, 32866-32877 (2018)

14. C. B. Martin, "Design issues of a hyperfield fisheye lens," in Optical Science and Technology, the SPIE 49th Annual Meeting (SPIE2004), p. 9

15. Meister, D., Sheedy, J.E.: Introduction to ophthalmic optics (SOLA Optical USA) (2000)

16. Kommnick, J., Schal, S., Fricke, V., Thape, T., Fischer, H.: Augenoptik in Lernfeldern (Handwerk + Technik GmbH) (2014)

17. Goersch, H.: Wörterbuch der Optometrie. DOZ-Verlag (2004)

18. Davidson, N., Friesem, A.A., Hasman, E.: Analytic design of hybrid diffractiverefractive achromats. Appl. Opt. 32, 4770-4774 (1993)

19. Zhou, G., Leung, H.M., Yu, H., Kumar, A.S., Chau, F.S.: Liquid tunable diffractive/refractive hybrid lens. Opt. Lett. 34, 2793-2795 (2009)

20. Li, L.: New formulation of the Fourier modal method for crossed surfacerelief gratings. J. Opt. Soc. Am. A. 14, 2758-2767 (1997)

21. Loewen, E.G., Popov, E.: Diffraction Gratings and Applications. Taylor \& Francis (1997)

22. Riedl, M.J.: Optical Design: Applying the Fundamentals. SPIE Press (2009)

23. DIN Deutsches Institut für Normung e. V., "Ophthalmic Optics - Uncut Finished Spectacle Lenses - Part 1: Specifications for Single-Vision and Multifocal Lenses (ISO 8980-1:2004)"; German version EN ISO 8980-1:2004,

24. Lee, C.-K., Wu, J.W.-J., Yeh, S.-L., Tu, C.-W., Han, Y.-A., Liao, E.H.-Z., Chang, L.Y.Y., Tsai, I.-E., Lin, H.-H., Hsieh, J.C.-T.: Optical configuration and colorrepresentation range of a variable-pitch dot matrix holographic printer. Appl. Opt. 39, 40-53 (2000)

25. Hong, K., Park, S.-g., Yeom, J., Kim, J., Chen, N., Pyun, K., Choi, C., Kim, S., An, J., Lee, H.-S.: Resolution enhancement of holographic printer using a hogel overlapping method. Opt. Express. 21, 14047-14055 (2013)

26. Su, J., Yan, X., Huang, Y., Jiang, X., Chen, Y., Zhang, T.: Progress in the synthetic holographic stereogram printing technique. Appl. Sci. 8, 851 (2018)

27. Marín-Sáez, J., Atencia, J., Chemisana, D., Collados, M.-V.: Characterization of volume holographic optical elements recorded in Bayfol HX photopolymer for solar photovoltaic applications. Opt. Express. 24, A720-A730 (2016)

28. Berneth, H., Bruder, F.-K., Fäcke, T., Jurbergs, D., Hagen, R., Hönel, D., Rölle, T., Walze, G.: Bayfol HX Photopolymer for Full-Color Transmission Volume Bragg Gratings, pp. 900602-900602-900610 (2014)

\section{Publisher's Note}

Springer Nature remains neutral with regard to jurisdictional claims in published maps and institutional affiliations.

\section{Submit your manuscript to a SpringerOpen ${ }^{\circ}$ journal and benefit from:}

- Convenient online submission

- Rigorous peer review

- Open access: articles freely available online

- High visibility within the field

- Retaining the copyright to your article

Submit your next manuscript at $>$ springeropen.com 